\title{
Role of Hypothalamic-Pituitary Axis in EGF Action on Maturation of Adrenal Gland in Fetal Rhesus Monkey In Vivo
}

\author{
CATHERINE L. COULTER, LEANNA C. READ, SEAN J. BARRY, ALICE F. TARANTAL, AND \\ DENNIS M. STYNE \\ Department of Physiology, University of Adelaide, Adelaide, SA, Australia, 5005 [C.L.C.]; Child Health \\ Research Institute, North Adelaide, SA, Australia 5006 [L.C.R.]; and Department of Pediatrics [S.J.B., \\ A.F.T., D.M.S.] and California Regional Primate Research Center [A.F.T.], University of California, \\ Davis, Davis, California 95616, U.S.A.
}

\begin{tabular}{|c|c|}
\hline \multicolumn{2}{|c|}{ ABSTRACT } \\
\hline 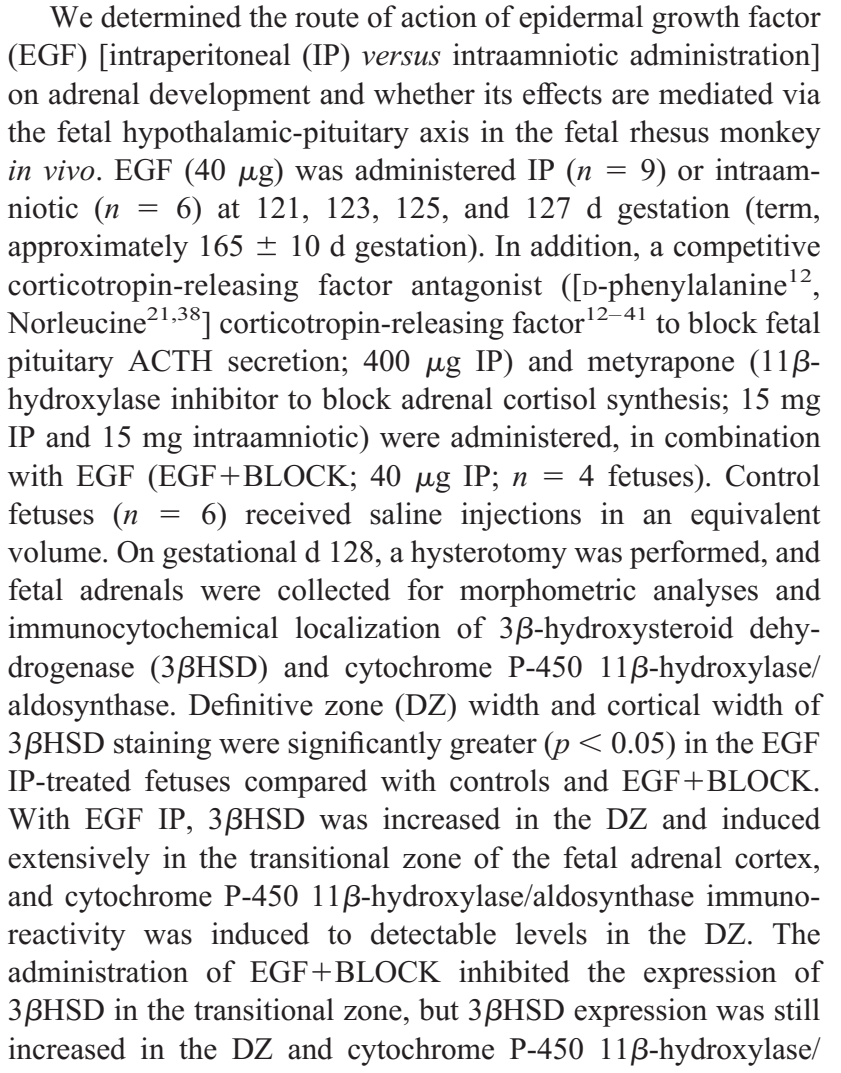 & 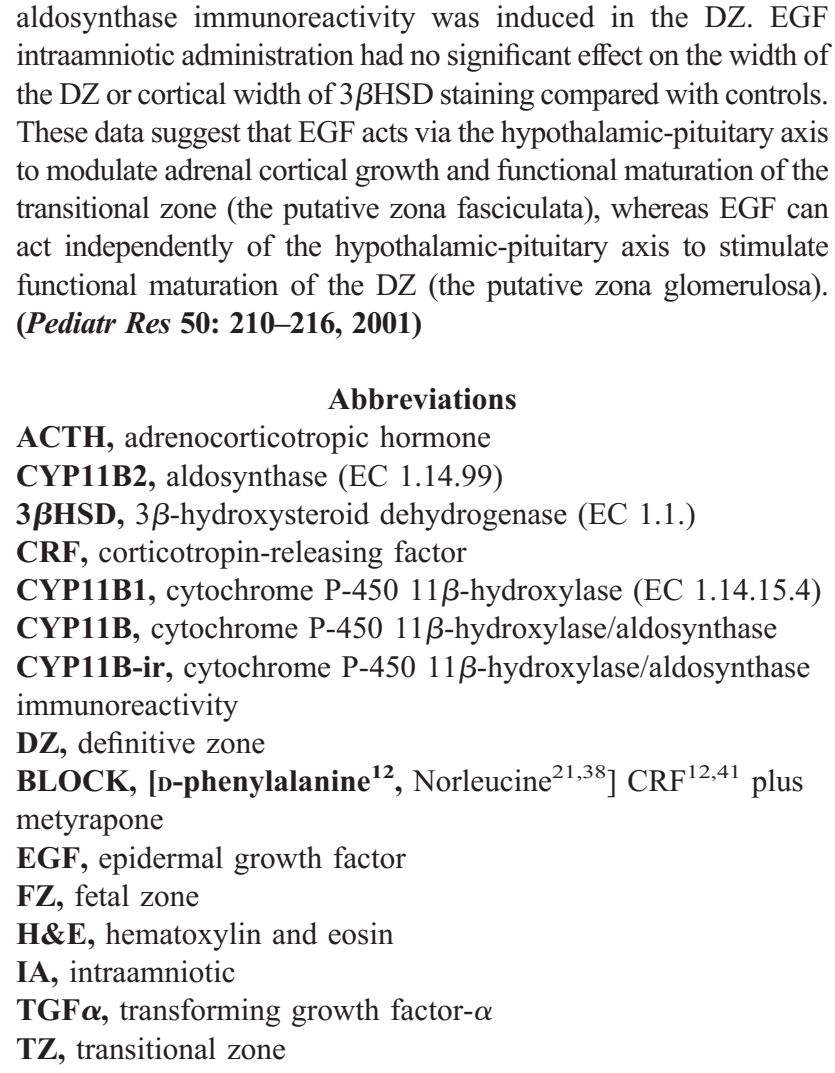 \\
\hline
\end{tabular}

The development of the steroidogenic capacity of the fetal adrenal gland is necessary for the maintenance of intrauterine

Received January 20, 2000; accepted January 17, 2001

Correspondence and reprint requests: Catherine L. Coulter, Ph.D., Department of Physiology, University of Adelaide, North Terrace, Adelaide, South Australia, Australia, 5005; e-mail: catherine.coulter@adelaide.edu.au

Supported by a Jean B. Reid Fellowship, the Faculty of Medicine, University of Adelaide (C.L.C.), the National Health and Medical Research Council of Australia (Grant homeostasis, development of enzyme systems necessary for extrauterine life, and, at least in some species, the timing of

990275 to C.L.C.), an NHMRC Block Grant to the Baker Medical Research Institute, and by NIH Grants HD17814, HD24959, and HD 08478.

Presented in part, at the $\mathrm{X}$ International Congress on Hormonal Steroids, Quebec, Canada, June 1998.

Current address (S.J.B.): The Rowe Program in Genetics, University of California, Davis, Davis, CA 95616, U.S.A. 
parturition. Secretion of cortisol from the fetal adrenal is necessary for the maturation of essential organ systems and plays a key role in the fetal response to intrauterine stress (1-5). After birth, the secretion of aldosterone from the newborn adrenal is a key component of the renin-angiotensin system and is essential for the maintenance of fluid balance and blood pressure during postnatal life. Previous studies in the human and nonhuman (rhesus monkey) primate fetus have indicated that the adrenal gland comprises three functional zones: 1) the large, inner FZ, which has the enzymes necessary for dehydroepiandrosterone sulfate production beginning early in gestation; 2) the TZ between the inner FZ and outer DZ, which possesses enzymes necessary for cortisol production beginning in late gestation; and 3) the outer, DZ, which appears to function as a reservoir of progenitor cells, which may populate the remainder of the gland, and does not acquire a steroidogenic phenotype with the capacity to produce mineralocorticoids until near term (1, 2, 6-13). Recently, we have shown that timing of the induction of $3 \beta \mathrm{HSD}$ in the TZ and CYP11B-ir in the DZ are coincident with increased circulating concentrations of cortisol and aldosterone in late gestation (12). These data suggest that functional maturation of the fetal adrenal cortex is dependent on the specific zonal expression of $3 \beta$ HSD and CYP11B, which are pivotal enzymes in adrenal steroidogenesis $(1,2)$. Thus, to understand the functional maturation of the primate fetal adrenal, it is important to determine the factors that regulate induction of $3 \beta \mathrm{HSD}$ and CYP11B expression.

Recently, we have demonstrated that administration of EGF into the fetal peritoneal and amniotic fluid cavities stimulated adrenal cortical growth and the expression of $3 \beta \mathrm{HSD}$ in the adrenal cortex of the fetal rhesus monkey in vivo (14). Studies have shown that EGF can stimulate the secretion of CRF from the hypothalamus and ACTH from the pituitary (15). The presence of specific receptors for EGF on both DZ and FZ cells has been demonstrated, and EGF stimulates proliferation of human fetal adrenal cells in vitro (16). It has also been shown that EGF stimulates secretion of cortisol and aldosterone as well as angiotensin II-induced aldosterone secretion from the adrenal gland (17). Thus, EGF may act both directly and indirectly via the fetal hypothalamic-pituitary axis to stimulate adrenal growth and steroidogenesis. In the rhesus monkey, amniotic fluid contains substantial amounts of a factor that binds to the EGF receptor in radioreceptor assays. This could reflect EGF or TGF $\alpha$, the latter having been identified in amniotic fluid in other studies. TGF $\alpha$ is a member of the EGF family and acts specifically via the EGF receptor (15). In these studies, we have used EGF, rather than $\operatorname{TGF} \alpha$, because it was made available to us as a recombinant protein in sufficient amounts for in vivo studies. To determine the route of action of EGF on adrenal development, in one group of fetuses we administered EGF IA and in a second group of fetuses we administered EGF directly into the fetus by the IP route. In addition, to determine whether the effects of EGF were directly on the adrenal gland or mediated via the hypothalamicpituitary axis, we administered EGF IP in combination with a competitive CRF antagonist (to block fetal pituitary ACTH secretion). In the present study, we examined the effects of
EGF administration and the mode of action of EGF on adrenal growth variables using morphometric techniques and determined the pattern of localization of $3 \beta \mathrm{HSD}$ and CYP $11 \mathrm{~B}$ in the fetal rhesus adrenal using immunocytochemical techniques.

\section{METHODS}

Animals and EGF administration. Twenty-five pregnant rhesus monkeys (Macaca mulatta) were obtained from the time-mated breeding colony at the California Regional Primate Research Center, University of California, Davis, CA, U.S.A. Animals were maintained in accordance with the National Institutes of Health Guide for the Care and Use of Animals, and the protocols used in this study were approved by the Institutional Animal Use and Care Committee at the University of California, Davis. Before IA or IP injections, the dams were administered ketamine hydrochloride $(10 \mathrm{mg} / \mathrm{kg})$, and EGF administration was performed with ultrasound guidance as described previously (18). Recombinant human EGF was provided by Chiron (Emeryville, CA, U.S.A.). EGF (40 $\mu \mathrm{g}$ in 1 $\mathrm{mL}$ of saline) was administered via the IP route $(n=9)$ or IA $(n=6)$ at $121,123,125$, and $127 \mathrm{~d}$ gestation (term, $165 \pm$ $10 \mathrm{~d}$ ). In four fetuses, a competitive CRF antagonist ([Dphenylalanine $^{12}$, Norleucine $\left.{ }^{21,38}\right] \mathrm{CRF}^{12-41}$ to block fetal pituitary ACTH secretion; $400 \mu \mathrm{g}$ IP) and metyrapone (CYP11B1 inhibitor to block adrenal cortisol synthesis; $15 \mathrm{mg}$ IP and $15 \mathrm{mg}$ IA) were also administered, in combination with EGF IP (EGF+BLOCK; $40 \mu \mathrm{g}$ ) at the same gestational times. The CRF antagonist was provided by Dr. Jean Rivier [The Salk Institute, San Diego, CA, U.S.A (19)], and the dose chosen was previously shown to be effective in the fetal rhesus monkey at modulating pituitary ACTH secretion by blocking the fetal hemorrhage-induced increase in fetal plasma ACTH concentrations $(1,20)$. Metyrapone treatment was included in the experimental paradigm, to determine whether the stimulatory effects of EGF treatment observed on fetal lung maturation (18, 21-23) were direct or mediated via the fetal adrenals. The data on the effects of EGF+BLOCK, EGF IP, or EGF IA on lung development are the subject of a separate publication (in preparation). In six control fetuses, saline was administered (1 $\mathrm{mL}$ of saline IP and $1 \mathrm{~mL}$ of saline IA). At $128 \mathrm{~d}$ gestation ( $78 \%$ of gestation), hysterotomies were performed, and fetal tissues were collected for analysis (see below), as described previously (18). Body and adrenal weights were obtained, and fetal adrenals were fixed in formalin (10\%) for morphologic and immunocytochemical analyses as described (14).

Immunocytochemistry. To localize $3 \beta \mathrm{HSD}$ and CYP11B protein in adrenals from fetal rhesus monkeys, we used a modification of the peroxidase/antiperoxidase method as described previously (14). A human placenta type-1 $3 \beta \mathrm{HSD}$ $(1: 200)$ polyclonal antibody was used and characterized previously (14) (generously provided by Dr. J. Ian Mason, Department of Clinical Biochemistry, University of Edinburgh, Scotland, U.K.). The CYP11B1/CYP11B2 (1:4000) polyclonal antibody was raised in rabbits against immunopurified ovine CYP11B enzyme and has been characterized previously (22, 24) and validated for use in primate adrenal tissue (12) (gen- 
erously provided by Professor Pieter Swart, Department of Biochemistry, University of Stellenboch, South Africa).

The ovine CYP11B antibody does not distinguish between $11 \beta$-hydroxylase and aldosterone synthase (24), which are encoded by separate but highly homologous genes, CYP11B1 and CYP11B2, respectively $(25,26)$, and therefore we have referred to this immunoreactive product as CYP11B-ir. Negative control sections were used in this study for all adrenals, in which the primary antisera was replaced with normal rabbit serum at dilutions equivalent to those for each antibody, i.e. $1: 200$ and 1:4000 for $3 \beta$ HSD and CYP11B, respectively. The immunocytochemical signal was visualized using 3,5 diaminobenzidine tetrahydrochloride, and the appearance of the light brown product was observed under a light microscope. To compare among the treatment groups, the same reaction time with 3,5 diaminobenzidine tetrahydrochloride was used for adrenal sections from control, EGF-treated, and $\mathrm{EGF}+\mathrm{BLOCK}$-treated fetuses.

Adrenal morphology and morphometry. Adrenal morphometric measurements and quantification of $3 \beta \mathrm{HSD}$ staining were made using a video-generated image analysis (Videometric 150, Oncor Inc, Gaithersburg, MD, U.S.A.) as described previously $(13,14)$, and assessments were conducted by an individual blinded to the treatment groups. In brief, the imageanalysis system permits the investigator to select the spectral composition of any desired color and allows one to establish limits of its true brilliance and intensity. These spectral values can be saved for equivalent measurements in successive sessions. The light microscope video-image is scanned automatically for the presence of the defined color, and the quantification (intensity) of $3 \beta \mathrm{HSD}$ staining (number of pixels) is presented as the number of grains per unit area of the image. In this study, the color limits were set manually to detect the brown immunocytochemical reaction product and to eliminate unstained background tissue. Triplicate measurements were made of the amount of $3 \beta$ HSD staining in a defined area $(20 \times$ $10^{3} \mu \mathrm{m}^{2}$ ) in three histologic sections from each adrenal gland from all fetuses and expressed as arbitrary units per square micrometer. Six measurements were made of the width of the DZ in H\&E-stained sections and of the width of cortical $3 \beta \mathrm{HSD}$ staining in sections of adrenals from control, EGFtreated, and EGF+BLOCK-treated animals.

Statistical analysis. The effect of EGF or EGF+BLOCK treatment on the adrenal to body weight ratio was compared using a one-way ANOVA. Comparisons of the effect of EGF or $\mathrm{EGF}+\mathrm{BLOCK}$ treatment on width of the DZ, cortical width of $3 \beta \mathrm{HSD}$ staining, and amount of $3 \beta \mathrm{HSD}$ staining were made using a two-way ANOVA with treatment (i.e. EGF IP, EGF IA, EGF+BLOCK, control) and replication. The two-way ANOVA tested for variation among morphometric measurements of the adrenal sections from one animal, among animals within a treatment group, and among control and treated groups. The Fisher's protected least square difference test was used after ANOVA to compare significant differences among mean values, and $p<0.05$ was considered to be significant. All data are expressed as mean \pm SEM. Data were analyzed using SuperANOVA (Abacus Concepts, Berkeley, CA,
U.S.A.) on a Macintosh personal computer (Apple Computer Inc, Cupertino, CA, U.S.A.).

\section{RESULTS}

Fetal body weight, adrenal weight, and adrenal growth. Fetal body weights did not significantly differ among the control (273.3 $\pm 19.6 \mathrm{~g})$, EGF IP (288.1 $\pm 26.2 \mathrm{~g})$, EGF IA $(296.5 \pm 35.9 \mathrm{~g})$, and EGF+BLOCK groups $(307.3 \pm 27.5 \mathrm{~g})$. Fetal adrenal weights were greater in the EGF IP group (278.5 $\pm 57.1 \mathrm{mg}$ ) when compared with the control group (188. $1 \pm$ $33.5 \mathrm{mg})$. In contrast, the fetal adrenal weights were not different among the EGF IA (240.8 $\pm 82.2 \mathrm{mg})$, $\mathrm{EGF}+\mathrm{BLOCK}(224.3 \pm 53.0 \mathrm{mg})$, and control fetuses. Similar data were found when examining the adrenal weight to body weight ratio. Adrenal to body weight ratios were significantly greater $(p<0.05)$ in the EGF IP group $(1.01 \pm 0.05 \mathrm{~g} / \mathrm{kg})$ compared with control fetuses $(0.69 \pm 0.05 \mathrm{~g} / \mathrm{kg}$; Fig. 1). In contrast, the adrenal to body weight ratios were not different among the EGF IA, EGF+BLOCK, and control fetuses (Fig. 1). The width of the DZ from EGF IP-treated fetuses was approximately twice the width of the DZ from control fetuses (Figs. 2 and 3, $A$ and $C$ ). In contrast, the width of the DZ was not significantly different among the EGF IA, EGF+BLOCK, and control fetuses (Figs. 2 and 3, $A, B$, and $D$ ).

Immunocytochemistry. The width of the DZ and cortical width of $3 \beta \mathrm{HSD}$ staining were significantly greater $(p<0.05)$ in the EGF IP group $(132 \pm 18 \mu \mathrm{m}, 329 \pm 25 \mu \mathrm{m})$ compared with the control group $(73 \pm 8 \mu \mathrm{m}, 141 \pm 9 \mu \mathrm{m})$ and EGF+BLOCK group $(69 \pm 3 \mu \mathrm{m}, 189 \pm 19 \mu \mathrm{m}$; Figs. 2 and $4)$. EGF IA administration had no significant effect $(p>0.05)$ on the width of the DZ or cortical width of $3 \beta \mathrm{HSD}$ staining $(106 \pm 10 \mu \mathrm{m}, 152 \pm 12 \mu \mathrm{m})$ compared with controls (Figs. 2 and 4).

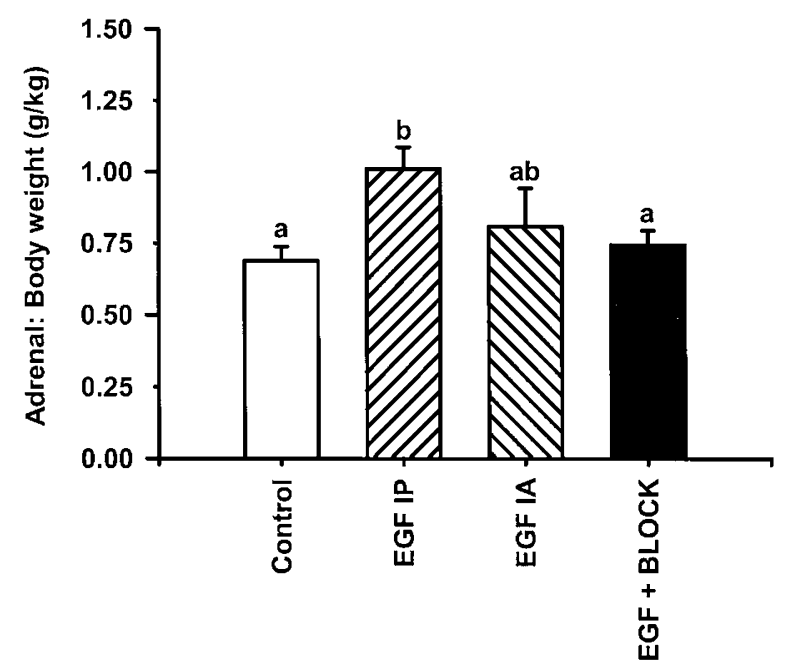

Figure 1. Adrenal to body weight ratio measurements from control ( $n=6$; open bars), EGF IP ( $n=9$; forward-angled hatched bars), EGF IA ( $n=6$; reverse-angled hatched bars), and EGF+BLOCK ( $n=4$; solid bars) rhesus monkey fetuses at $128 \mathrm{~d}$ gestation. Data are expressed as mean \pm SEM. A one-way ANOVA was used to compare the control and treated animals, and $p$ $<0.05$ was considered significant. Groups designated with the same letter are not significantly different from each other, whereas groups designated with different letters are significantly different from each other. 


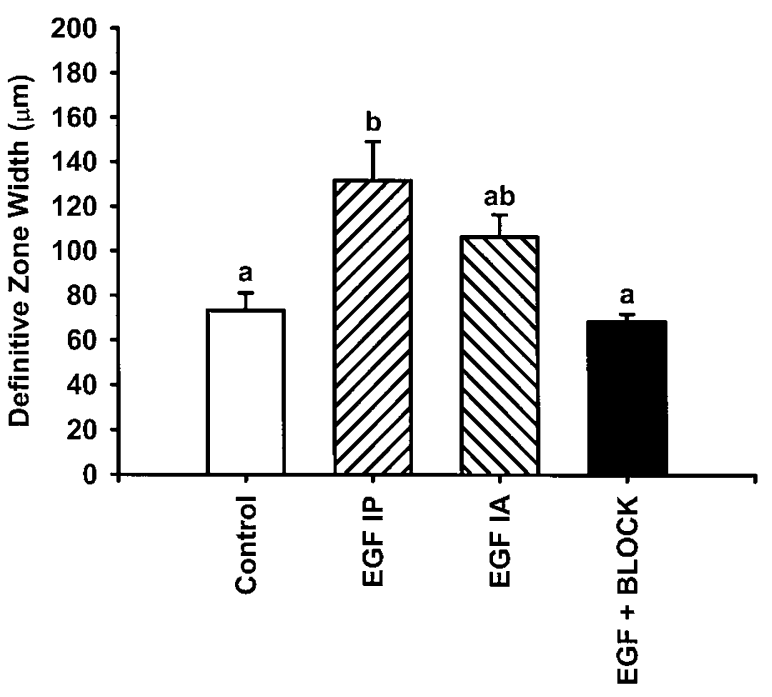

Figure 2. An image-analysis system was used to measure the DZ width on H\&E-stained adrenal glands from control $(n=6$; open bars), EGF IP $(n=9$; forward-angled hatched bars), EGF IA ( $n=6$; reverse-angled hatched bars), and EGF+BLOCK $(n=4$; solid bars) rhesus monkey fetuses at $128 \mathrm{~d}$ gestation. For each animal, a mean of six measurements of the width of the DZ was made, and the data are expressed as mean \pm SEM for control and treated fetuses. A two-way ANOVA with treatment and replication as factors was used to compare the control and treated animals, and $p<0.05$ was considered significant. Groups designated with the same letter are not significantly different from each other, whereas groups designated with different letters are significantly different from each other.

In the control animals, $3 \beta \mathrm{HSD}$ immunostaining was present in the $\mathrm{DZ}$ and did not extend greatly into the $\mathrm{TZ}$. In fetuses that received EGF IA alone or EGF+BLOCK, $3 \beta \mathrm{HSD}$ immunostaining was also present in the $\mathrm{DZ}$ and a small $\mathrm{TZ}$ was present (Figs. 4 and $5, A, B$, and $D$ ). However, the intensity of staining for $3 \beta \mathrm{HSD}$ protein in the DZ was increased in the EGF+BLOCK group when compared with the control group (Figs. $5 D$ and 6). In contrast to control animals, there was abundant $3 \beta \mathrm{HSD}$ immunostaining in the DZ, and $3 \beta \mathrm{HSD}$ was induced extensively in the TZ of the fetal adrenal after administration of EGF IP alone (Figs. 4, 5B, and 6). In parallel with these observations on $3 \beta \mathrm{HSD}$ localization, the mean width of cortical $3 \beta$ HSD staining was significantly greater $(p<0.05)$ in

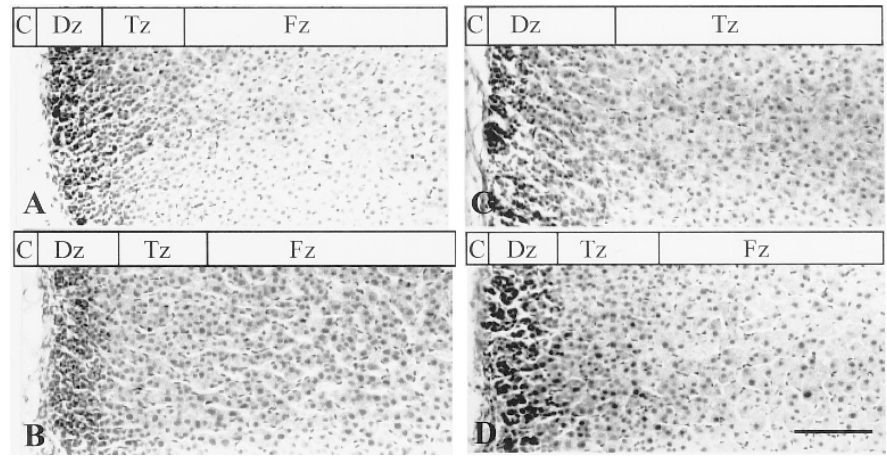

Figure 3. Photomicrographs of H\&E-stained sections $(8 \mu \mathrm{m})$ of adrenal glands from control $(A)$, EGF IA $(B)$, EGF IP $(C)$, and EGF+BLOCK $(D)$ rhesus monkey fetuses at $128 \mathrm{~d}$ gestation. The open bar across the top of each panel identifies the region directly underneath, namely, the DZ, TZ, FZ, and adrenal capsule (C). The scale bar represents $150 \mu \mathrm{m}$.

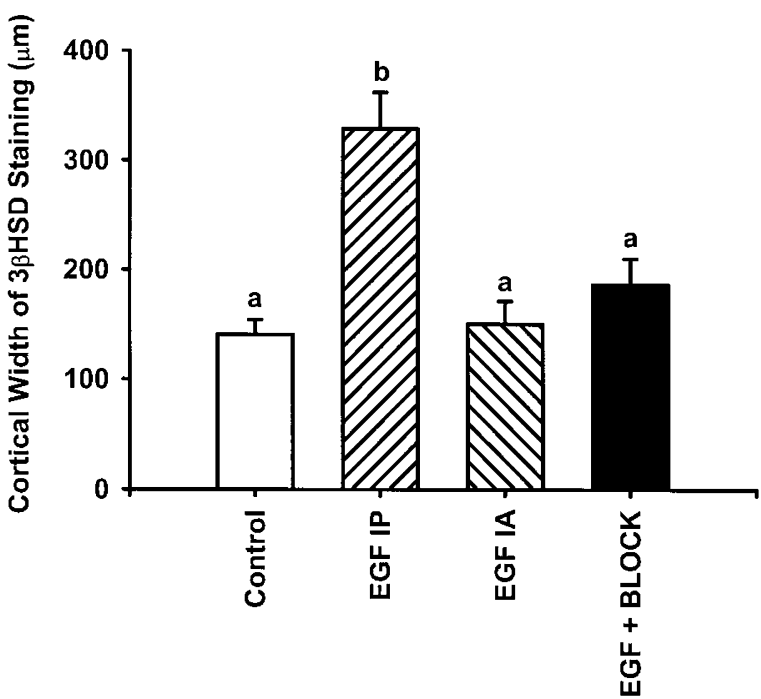

Figure 4. The cortical width of $3 \beta \mathrm{HSD}$ staining of adrenal glands from control ( $n=6$; open bars), EGF IP ( $n=9$; forward-angled hatched bars), EGF IA $(n=6$; reverse-angled hatched bars $)$, and EGF+BLOCK $(n=4$; solid bars) rhesus monkey fetuses at $128 \mathrm{~d}$ gestation. For each animal, six measurements of the cortical width of $3 \beta \mathrm{HSD}$ staining were made using an imageanalysis system. A two-way ANOVA with treatment and replication as factors was used to compare the control and treated animals, and $p<0.05$ was considered significant. Groups designated with the same letter are not significantly different from each other, whereas groups designated with different letters are significantly different from each other. Data are expressed as mean \pm SEM.

the EGF IP-treated fetuses compared with control, EGF IA, and EGF + BLOCK fetuses, as determined by image analysis (Fig. 4).

It should be noted in the EGF IA fetuses that although there was a trend for an increase in the adrenal to body weight ratio (Fig. 1) and width of the DZ (Fig. 2), this was not paralleled by any significant change in cortical width (Fig. 4), the amount (Fig. 6) of $3 \beta \mathrm{HSD}$ staining, or the pattern of localization of $3 \beta$ HSD (Fig. $5 B$ ).

In control and EGF IA animals, CYP11B-ir was present in the $\mathrm{TZ}$ and FZ, but was not detectable in the DZ (Fig. 5, $E$ and $G)$. EGF administration into the IP cavity induced CYP11B-ir in the DZ (Fig. 5I). However, the effect of EGF IP on staining for CYP11B in the DZ was not blocked by coadministration with the CRF antagonist and metyrapone (Fig. $5 K$ ). Although CYP11B-ir was detected in the FZ cells, it was not detectable in adrenomedullary cells in any of the treatment groups (Fig. 5, $F, H, J$, and $L$ ). No staining was detected when the primary antisera were replaced with normal rabbit serum (data not shown).

\section{DISCUSSION}

In the fetal rhesus monkey in vivo, adrenal cortical growth and functional maturation of the adrenal gland are stimulated by EGF administration into the peritoneal and amniotic fluid cavities (14). In the present study, to extend these observations, we have determined the route of action of EGF on the fetal adrenal. We have found that EGF administered via the IP route, but not the IA route, significantly stimulated adrenal cortical 


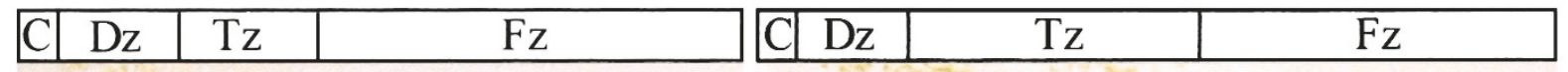

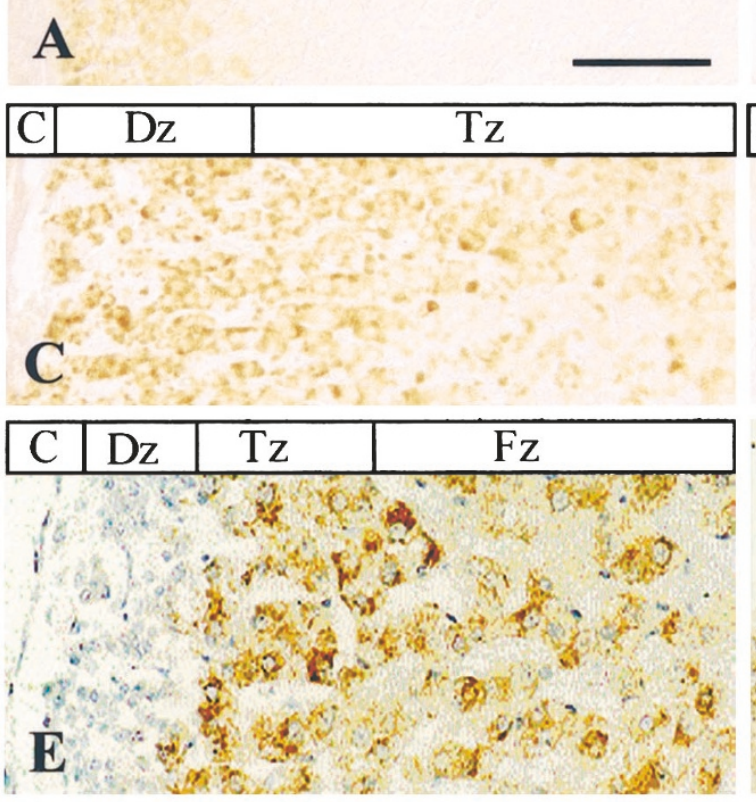

B

is.
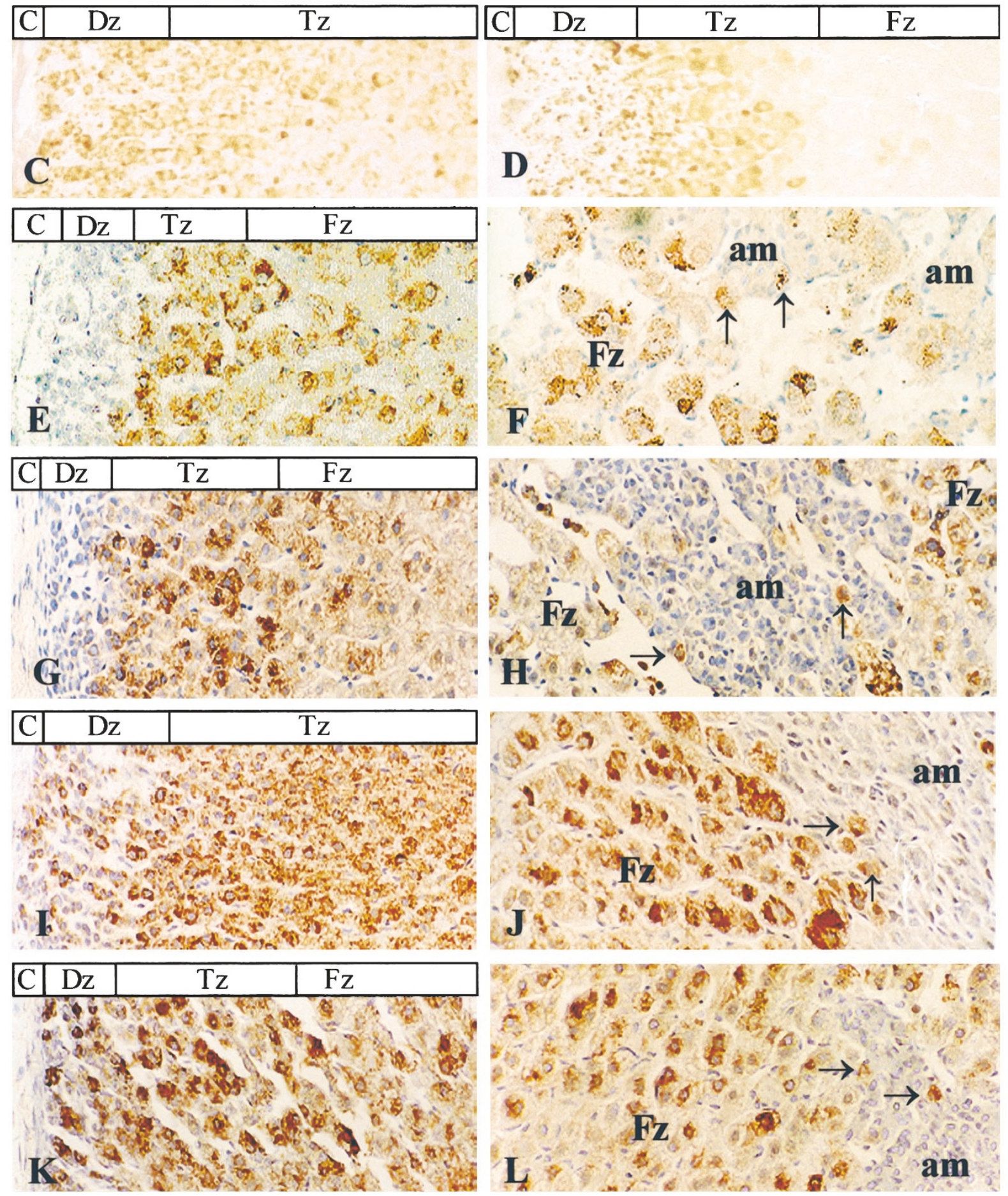

Figure 5. Photomicrographs of $8-\mu \mathrm{m}$ sections of $128 \mathrm{~d}$ gestation rhesus monkey fetal adrenal glands from control $(A)$, EGF IA $(B)$, EGF IP $(C)$, and EGF+BLOCK $(D)$ fetuses, stained for $3 \beta \mathrm{HSD}$, and control $(E, F)$, EGF IA $(G, H)$, EGF IP $(I, J)$, and EGF+BLOCK $(K, L)$ fetuses, stained for CYP11B-ir, in which brown staining identifies adrenocortical cells that are immunopositive for $3 \beta \mathrm{HSD}$ or CYP11B-ir, respectively. The sections stained for CYP11B-ir are counterstained with hematoxylin to identify cell nuclei (blue). For panels $A-E, G, I$, and $K$, the open bar across the top of each panel identifies the region immediately underneath, namely, the DZ, TZ, FZ, and adrenal capsule (C). Panels $F, H, J$, and $L$ are photomicrographs of the central corticomedullary region, in which the adrenomedullary cells interdigitate with FZ cells of the adrenal cortex. The arrows identify CYP11B-ir FZ cells adjacent to or within the unstained adrenomedullary $(A M)$ area. The scale bar represents $150 \mu \mathrm{m}$. 


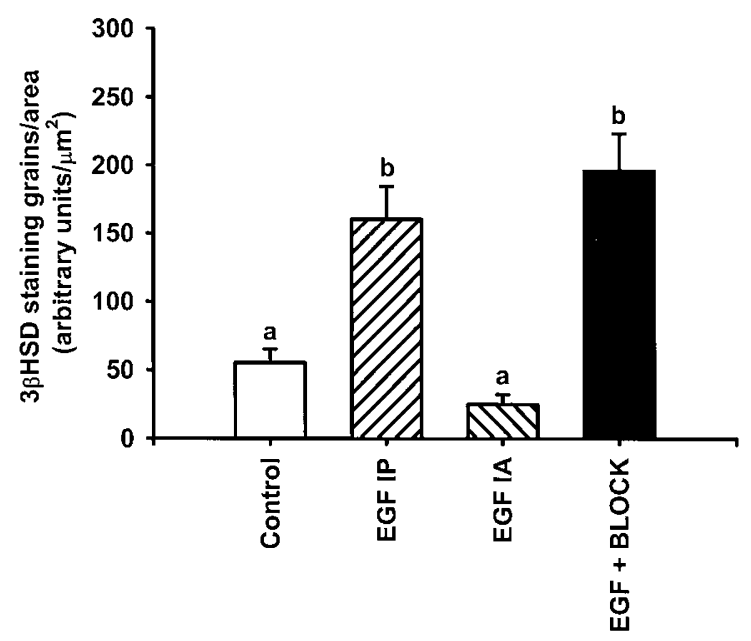

Figure 6. The amount of $3 \beta \mathrm{HSD}$ staining (arbitrary units per square micrometer) from adrenal glands from control ( $n=6$; open bars), EGF IP ( $n=9$; forwardangled hatched bars), EGF IA ( $n=6$; reverse-angled hatched bars), and $\mathrm{EGF}+\mathrm{BLOCK}(n=4$; solid bars) rhesus monkey fetuses at $128 \mathrm{~d}$ gestation. For each animal, triplicate measurements of the $3 \beta$ HSD staining were made using an image-analysis system. A two-way ANOVA with treatment and replication as factors was used to compare the control and treated animals, and $p<0.05$ was considered significant. Groups designated with the same letter are not significantly different from each other, whereas groups designated with different letters are significantly different from each other. Data are expressed as mean \pm SEM.

growth. EGF receptors are present on human fetal adrenal cells, and EGF stimulates the proliferation of these cells in vitro (16); therefore, we postulated that this may be a direct action of EGF on adrenal cells (14). Alternatively, EGF has been shown to increase both CRF and ACTH release (15) and potentiate ACTH-mediated steroidogenesis in the human fetal adrenal (27); therefore, EGF may mediate its effects on the adrenal gland by activation or modulation of the hypothalamicpituitary axis.

To provide further insight into the mechanism of action of EGF on adrenal development and to assess the role of the fetal hypothalamic-pituitary axis in mediating the effects of EGF on adrenal growth, we administered the competitive CRF antagonist [D-phenylalanine ${ }^{12}$, Norleucine $\left.{ }^{21,38}\right]_{\mathrm{CRF}^{12-41}}$ in combination with EGF. We found that the effects of EGF on increasing adrenal weight and stimulating the growth of the DZ were blocked by the administration of the CRF antagonist. These data suggest that EGF mediates its effects on adrenal growth not by a direct action on the fetal adrenal but indirectly via the fetal hypothalamic-pituitary axis. It is clear that from previous studies that this CRF antagonist is effective in the fetal rhesus monkey at modulating pituitary ACTH secretion as it has been shown to block the fetal hemorrhage-induced increase in fetal plasma ACTH concentrations $(1,20)$. We also note that the CRF antagonist was able to block the secondary effects of metyrapone. Recently, we have shown that metyrapone treatment alone dramatically stimulated adrenal cortical growth (28). Metyrapone blocks cortisol synthesis by inhibiting CYP11B1 activity and thus removes the negative feedback effect of cortisol to activate the fetal hypothalamic-pituitary axis and stimulate adrenal growth. These findings provide further evidence to demonstrate the effectiveness of the CRF antagonist in blocking fetal pituitary ACTH secretion and also suggest that EGF acts via the fetal hypothalamic-pituitary axis to stimulate adrenal cortical growth. However, in the present study we did not measure fetal plasma ACTH concentrations.

The effects of EGF administration on stimulating the premature morphologic maturation of the primate fetal adrenal are paralleled by the stimulation of the functional maturation of the adrenal gland. The induction of CYP11B protein in the DZ and $3 \beta \mathrm{HSD}$ protein in the $\mathrm{TZ}$ of the fetal adrenal are markers for the functional differentiation of the fetal into the adult cortex (9, 12, 13). Between 110 and $150 \mathrm{~d}$ of gestation, CYP11B is localized predominantly in the TZ and $3 \beta \mathrm{HSD}$ is localized predominantly in the DZ, whereas after $155 \mathrm{~d}$ gestation, CYP11B and $3 \beta \mathrm{HSD}$ are localized extensively in both the DZ and $\mathrm{TZ}$ of the fetal primate adrenal gland $(12,13)$. The premature expression of CYP11B in the DZ and $3 \beta \mathrm{HSD}$ in the $\mathrm{TZ}$ can be induced by increasing fetal pituitary $\mathrm{ACTH}(12,13)$. We also found that the IP and IA administration of EGF stimulated an increase in adrenal content of $3 \beta \mathrm{HSD}$ protein primarily by inducing expression of $3 \beta \mathrm{HSD}$ protein in the TZ (14). In the current study, the administration of EGF via the IP route alone was sufficient to stimulate the functional maturation of the fetal adrenal as indicated by the premature induction of expression of CYP11B protein in the DZ and $3 \beta \mathrm{HSD}$ protein in the TZ. However, if EGF was administered via the IA route alone, there was no change in the pattern of localization of either CYP11B or $3 \beta \mathrm{HSD}$, consistent with the lack of a significant effect of EGF IA administration on adrenal weight and DZ growth measurements. These data indicate that the effective route of action of EGF on adrenal gland growth and functional maturation is achieved via the IP route alone but not via the IA route.

The CRF antagonist successfully inhibited the EGFactivated induction of $3 \beta \mathrm{HSD}$ in the TZ. These data suggest that the effects of EGF on functional maturation and induction of $3 \beta \mathrm{HSD}$ are also mediated via the hypothalamic-pituitary axis. It is interesting that whereas administration of the competitive CRF antagonist blocked the effects of EGF on inducing $3 \beta \mathrm{HSD}$ in the TZ, we still observed increased $3 \beta \mathrm{HSD}$ staining and induction of CYP11B-ir in the DZ, which is the putative zona glomerulosa of the adult adrenal gland. We know from studies in the adult adrenal gland that the zona glomerulosa and zona fasciculata are differentially regulated via angiotensin and ACTH, respectively. Our data suggest that in the fetal adrenal gland there may be a direct action of EGF on the DZ. Previous studies have shown that EGF receptors are present on human fetal adrenal cells (16) and that EGF stimulates aldosterone secretion from the zona glomerulosa (29). Alternatively EGF may modulate its effects via angiotensin II, as EGF has been shown to potentiate angiotensin II-induced aldosterone secretion (29). Clearly, the role of EGF in mediating functional maturation of the DZ requires further investigation.

In summary, we have shown that IP administration of EGF is effective in stimulating the growth and functional maturation of the fetal primate adrenal gland. Administration of a competitive CRF antagonist, [D-phenylalanine ${ }^{12}$, Norleucine $^{21,38}$ ] $\mathrm{CRF}^{12-41}$, blocked the action of EGF on stimulating adrenal 
growth and the induction of $3 \beta \mathrm{HSD}$ in the TZ but did not diminish the increase in $3 \beta \mathrm{HSD}$ staining or induction of CYP11B in the DZ. These data suggest that EGF acts via the hypothalamic-pituitary axis to modulate the growth of the DZ (the putative zona glomerulosa) and growth and functional maturation of the TZ (the putative zona fasciculata), whereas EGF acts independently of the hypothalamic-pituitary axis to stimulate functional maturation of the DZ.

Acknowledgments. The authors thank Dr. Jean Rivier, The Salk Institute, San Diego, CA, U.S.A., for the generous gift of CRF antagonist, Dr. Carlos George-Nascimento, Chiron Corp, Emeryville, CA, U.S.A., for the EGF peptide, Dr. J. Ian Mason, University of Edinburgh, Scotland, U.K., for the $3 \beta \mathrm{HSD}$ antibody, and Professor Pieter Swart, University of Stellenboch, South Africa, for the CYP11B antibody. We thank Drs. Bill Lasley and Paul Goldsmith for their interest and contribution to this project, and Cheryl Malcamp for her expert technical assistance.

\section{REFERENCES}

1. Jaffe RB, Mulchahey JJ, DiBlasio AM, Martin MC, Blumenfeld Z, Dumesic DA 1988 Peptide regulation of pituitary and target tissue function and growth in the primate fetus. Rec Prog Horm Res 44:431-549

2. Pepe GJ, Albrecht ED 1990 Regulation of primate fetal adrenal cortex. Endocr Rev 11:151-176

3. Liggins GC 1976 Adrenocortical-related maturational events in the fetus. Am J Obstet Gynecol 126:931-941

4. Challis JRG, Brooks AN 1989 Maturation and activation of hypothalamic-pituitaryadrenal function in fetal sheep. Endocr Rev 10:182-204

5. Ballard P 1986 Hormones and lung maturation. Monogr Endocrinol 28:1-354

6. Sholl S 1982 Corticoid formation by the monkey fetal adrenal: evaluation of 17-, 21 and 11-hydroxlyase activities. Steroids 40:475-484

7. Doody KM, Carr BR, Rainey WE, Byrd W, Murray BA, Strickler RC, Thomas JL, Mason JI 19903 Beta-hydroxysteroid dehydrogenase/isomerase in the fetal zone and neocortex of the human fetal adrenal gland. Endocrinology 126:2487-2492

8. Voutilainen R, Ilveskmaki V, Miettinen P 1991 Low expression of $3 \beta$-hydroxy-5-ene steroid dehydrogenase gene in the human fetal adrenals in vivo: adrenocorticotropin and protein-kinase $\mathrm{C}$-dependent regulation in adrenocortical cultures. J Clin Endocrinol Metab 72:761-767

9. Mesiano S, Coulter CL, Jaffe RB 1993 Localization of cytochrome P450 cholesterol side chain cleavage, cytochrome P450 17 $\alpha$-hydroxylase/17,20-lyase, and $3 \beta$ hydroxysteroid dehydrogenase/isomerase steroidogenic enzymes in the human and rhesus monkey fetal adrenal gland: reappraisal of functional zonation. J Clin Endocrinol Metab 77:1184-1189

10. Parker Jr CR, Faye-Petersen O, Stankovic AK, Mason JI, Grizzle WE 1995 Immunocytochemical evaluation of the cellular localization and ontogeny of $3 \beta$ hydroxysteroid dehydrogenase/delta 5-4 isomerase in human fetal adrenal glands. Endocr Res $21: 69-80$
11. Sholl SA 1983 Patterns of $3 \beta$-hydroxysteroid dehydrogenase delta ${ }^{5-4}$ isomerase activity in the rhesus monkey placenta and fetal adrenal. Steroids 41:769-776

12. Coulter CL, Jaffe RB 1998 Functional maturation of the primate fetal adrenal in vivo: 3. Specific zonal localization and developmental regulation of CYP21A2 (P450c21) and CYP11B1/CYP11B2 (P450c11/aldosterone synthase) lead to integrated concept of zonal and temporal steroid biosynthesis. Endocrinology 139:5144-5150

13. Coulter CL, Goldsmith PC, Mesiano S, Voytek CC, Martin MC, Mason JI, Jaffe RB 1996 Functional maturation of the primate fetal adrenal in vivo. II. Ontogeny of corticosteroid synthesis is dependent upon specific zonal expression of 3 betahydroxysteroid dehydrogenase/isomerase. Endocrinology 137:4953-4959

14. Coulter CL, Read LC, Carr BR, Tarantal AF, Barry S, Styne DM 1996 A role for epidermal growth factor in the morphological and functional maturation of the adrenal gland in the fetal rhesus monkey in vivo. J Clin Endocrinol Metab 81:1254-1260

15. Fisher DA, Lakshmanan J 1990 Metabolism and effects of epidermal growth factor and related factors in mammals. Endocr Rev 11:418-442

16. Crickard K, Jaffe RB 1981 Control of proliferation of human fetal adrenal cells in vitro. J Clin Endocrinol Metab 53:790-796

17. Thorburn GD, Waters MJ, Young IR, Dolling M, Buntine D, Hopkins PS 1981 Epidermal growth factor: a critical factor in fetal maturation? Ciba Found Symp 86:172-198

18. Plopper CG, St. George JA, Read LC, Nisho SJ, Weir AJ, Edwards L, Tarantal AF, Pinkerton KE, Merritt TA, Whitsett JA, George-Nascimento C, Styne DM 1992 Acceleration of alveolar type II cell differentiation in fetal rhesus monkey lung by administration of EGF. Am J Physiol 262:L313-L321

19. Gulyas J, Rivier C, Perrin M, Koerber SC, Sutton S, Corrigan A, Lahrichi SL, Craig AG, Vale W, Rivier J 1995 Potent, structurally constrained agonists and competitive antagonists of corticotropin-releasing factor. Proc Natl Acad Sci USA 92:1057510579

20. Coulter CL, Martin MC, Voytek CC, Hofmann JI, Jaffe RB 1993 Response to hemorrhagic stress in the rhesus monkey fetus in utero: effects on the pituitaryadrenal axis. J Clin Endocrinol Metab 76:1234-1240

21. Goetzman BW, Read LC, Plopper CG, Tarantal AF, George-Nascimento C, Merritt TA, Whitsett JA, Styne D 1994 Prenatal exposure to epidermal growth factor attenuates respiratory distress syndrome in rhesus infants. Pediatr Res 35:30-36

22. Edwards LA, Read LC, Nishio SJ, Weir AJ, Hull W, Barry S, Styne D, Whitsett JA, Tarantal AF, George-Nascimento C, Plopper CG 1995 Comparison of the distinct effects of epidermal growth factor and betamethasone on the morphogenesis of the gas exchange region and differentiation of alveolar type II cells in lungs of fetal rhesus monkeys. J Pharmacol Exp Ther 274:1025-1032

23. St. George JA, Read LC, Cranz DL, Tarantal AF, George-Nascimento C, Plopper CG 1991 Effect of epidermal growth factor on the fetal development of the tracheobronchial secretory apparatus in rhesus monkey. Am J Respir Cell Mol Biol 4:95-101

24. Bellstedt DU, Louv RP, Parringer BA, Werder N, VanderMerwe KJ, Swart P 1993 Antibody production to adrenal cytochrome p450-dependent enzymes using acidtreated bacteria as immune carriers. Biochem Soc Trans 21:414S

25. Kawamoto T, Mitsuichi V, Toda K, Yokoyama Y, Miyahara K, Miura S, Ohnishi T, Ichikawa Y, Nakao K, Imura H, Ulick S, Shiauta Y 1992 Role of steroid $11 \beta$ hydroxylase and steroid 18-hydroxylase in the biosynthesis of glucocorticoids and mineralocorticoids in humans. Proc Natl Acad Sci USA 89:1458-1462

26. Mornet E, Dupont J, Vitek A, White PC 1989 Characterization of two genes encoding human steroid 11 $\beta$-hydroxylase (P-45011B). J Biol Chem 264:20961-20967

27. Mesiano S, Jaffe RB 1997 Developmental and functional biology of the primate fetal adrenal cortex. Endocr Rev 18:378-403

28. Coulter CL, Goldsmith PC, Mesiano S, Voytek CC, Martin MC, Han VK, Jaffe RB 1996 Functional maturation of the primate fetal adrenal in vivo: I. Role of insulin-like growth factors (IGFs), IGF-I receptor, and IGF binding proteins in growth regulation. Endocrinology 137:4487-4498

29. Natarajan R, Nadler J 1991 Angiotensin II-induced aldosterone synthesis is potentiated by epidermal growth factor. Endocrinology 128:2285-2290 